\title{
Aplicación de la Metodología de Fusión de Imágenes Multidirección-Multiresolución (MDMR) a la Estimación de la Turbidez en Lagos
}

\section{Mario F. Lillo-Saavedra ${ }^{*_{1}}$ y Consuelo Gonzalo ${ }^{2}$}

(1) Universidad de Concepción, Facultad de Ingeniería Agrícola, Departamento de Mecanización y Energía, Avda. Vicente Méndez 595, Casilla 537, Chillán-Chile (e-mail: malillo@udec.cl) (2) Universidad Politécnica de Madrid, Facultad de Informática, Campus Montegancedo, 28660 Boadilla del Monte, Madrid-España (e-mail: chelo@fi.upm.es)

*autor a quien debe ser dirigida la correspondencia

\begin{abstract}
Resumen
Se propone mejorar la precisión en la estimación de características representativas de la calidad de las aguas de un lago mediante el uso de imágenes de satélite fusionadas. Las imágenes satelitales fuente han sido capturadas por los sensores a bordo del satélite Landsat 7. Las imágenes fusionadas se han obtenido mediante una nueva metodología de fusión, conceptualmente inspirada en una transformada multidirección-multirresolución (MDMR) y la transformada de ondículas calculada mediante el algoritmo de cavidades (Wavelet à trous), utilizando un banco de filtros direccionales y separables. La principal característica de esta metodología de fusión es el mecanismo de control de la calidad de las imágenes fusionadas. Los resultados muestran una notable mejora en la estimación de la calidad de las aguas del lago.
\end{abstract}

Palabras clave: fusión de imágenes, MDMR, transformada de ondículas, algoritmo de cavidades, calidad de aguas, turbidez

\section{Multidirection-Multiresolution Fusion Images Methology (MDMR) Applied to Turbidity Lake Estimation}

\begin{abstract}
It is proposed to improve the precision estimation of the representative characteristics of the quality characteristics of waters of a lake through the use of fused images. For that, images captured by the sensors of the Landsat 7 satellite have been used. The fused images have been obtained by means of a new fusion methodology, conceptually inspired by the Multidirection-Multiresolution Fusion Images Methology (MDMR) and by the Wavelet à trous algorithm, using a directional and separable filter bank. The main characteristic of this approach is the fused images quality control mechanism. The results show a noticeable improving in the reliability of the lake water quality estimation.
\end{abstract}

Keywords: image fusion, MDMR, wavelet, à trous algorithm, water quality, water turbidity 


\section{INTRODUCCIÓN}

En el ámbito de la teledetección, existe un amplio rango de fenómenos a estudiar que requieren disponer de imágenes de satélite que combinen una alta resolución espacial y espectral. En este sentido, algunas de las aplicaciones que pueden verse mas beneficiadas, son aquéllas donde la variabilidad espacial de los fenómenos estudiados sea un factor crítico en la precisión de los resultados obtenidos, como son, a modo de ejemplo, la generación de mapas temáticos, la detección de cambios o la monitorización y gestión de recursos naturales de forma general y de recursos hídricos de forma particular.

Una manera de disponer de imágenes de alta resolución tanto espacial como espectral, a costos accesibles, es la utilización de técnicas de fusión de imágenes. El concepto de fusión imágenes se puede entender como la combinación sinérgica de información proporcionada por varios sensores o por el mismo sensor en diferentes escenarios (espaciales, espectrales y temporales) (Lillo-Saavedra et al., 2006). Desde el punto de vista de la fusión de imágenes Multiespectrales (MULTI) y Pancromáticas (PAN), que se va a tratar en este trabajo, consiste en combinar de la forma más coherente posible, la información espectral de la imagen MULTI, con la espacial de la imagen PAN, con objeto de que la calidad global (espacial-espectral) de la imagen fusionada sea muy alta. La consecución de este objetivo, ha llevado a los investigadores del área, a la búsqueda de nuevas representaciones de imágenes que mejorasen la calidad de las imágenes fusionadas (Do y Vetterli, 2005; Lillo-Saavedra y Gonzalo, 2007).

Actualmente, el análisis multirresolución es una de las herramientas más utilizadas en las estrategias de fusión de imágenes, dada su capacidad de minimizar la incertidumbre intrínseca de los dominios de representación conjunta de la información. La Transformada Discreta Wavelet (TDW) se puede considerar como la aproximación más popular (Wald, 2002).

La principal imitación de la TDW reside en su baja anisotropía, que impide una selección precisa de los detalles extraídos de la imagen PAN y asociados a las altas frecuencias espaciales, minimizando la cantidad de información espectral que esta aporta a la imagen fusionada, por otro lado, maximizando la calidad espectral de la imagen MULTI degradada, asociada a las bajas frecuencias espaciales. En este sentido, se han llevado a cabo diferentes aproximaciones como las propuestas por Garzelli et al. (2004) y Lillo-Saavedra y Gonzalo (2006), que son dos versiones de la metodología de fusión mediante el algoritmo à trous (Nuñez et al., 1999), en las cuales se ponderan de dos maneras diferentes la cantidad de información procedente de la PAN que se integra en la MULTI. Una breve descripción de distintos algoritmos de fusión se ha incluido en la siguiente sección, pudiéndose encontrar descripciones más exhaustivas de los diferentes métodos en las referencias citadas.

En este contexto, surgió la necesidad de proponer un nuevo método de fusión altamente anisotrópico y redundante, que permitiera una selección adecuada de la información extraída desde las imágenes fuente. Es así que Gonzalo y Lillo-Saavedra (2007) propusieron un nuevo método de fusión, aplicado a imágenes Ikonos, que incluye ambos aspectos y el cual se basa en una representación multirresolución y multidirección (MDMR).

El objetivo central de este trabajo es mostrar la mejora en la estimación de turbidez de un cuerpo de agua, que proporciona la disponibilidad de imágenes de mejor calidad espacial y espectral (imágenes fusionadas) que las imágenes multiespectrales originales para el caso particular del sensor ETM+ del satélite Landsat 7.

\section{FUSION DE IMÁGENES ÓPTICAS}

En la actualidad se dispone de un gran número de metodologías y algoritmos para la fusión de imágenes ópticas, siendo las más utilizadas las basadas en diferentes transformadas. Algunas son muy sencillas desde un punto de vista conceptual, como las metodologías basadas en la transformada de Brovey (TB), el Análisis de Componentes Principales o la transformada IHS (intensidad, brillo y saturación) (Wald, 2002). Esta última cuenta con una amplia aceptación en las 
áreas de procesamiento de imágenes debido a su simplicidad conceptual. De hecho está incluida en diferentes paquetes de software comercial y se han desarrollado diferentes versiones mejoradas. Sin embargo, y como se demuestra en diferentes trabajos (Tu et al., 2001 y Choi, 2006), estas metodologías proporciona imágenes fusionadas cuyo color presenta considerables distorsiones respecto al color de las imágenes multiespectrales originales, esto impide su uso en diferentes tareas habituales en el área de teledetección, como son: la clasificación de imágenes o la detección de cambios. Otra limitación de este método es que solo se puede aplicar simultáneamente a 3 bandas. Un esquema del proceso de fusión mediante esta transformada se ha representado en la figura 1 a).

Por otro lado existe un elevado número de métodos basado en técnicas de análisis multirresolución, que utilizan fundamentalmente la Transformada Discreta Wavelet (TDW), tanto en su versión piramidal, mediante el algoritmo de Mallat (Mallat, 1999; González-Audícana et al., 2004), como en su versión redundante, utilizando el algoritmo à trous ("with holes") (Nuñez et al., 1999; GonzalezAudícana et al., 2005; Lillo-Saavedra y Gonzalo, 2006). La figura 1 b) muestra un esquema de fusión utilizando la TDW, empleando el algoritmo de Mallat. En general estos métodos proporcionan una mínima distorsión del color de las imágenes fusionadas, respecto de la imagen multiespectral, superior a las técnicas descritas más arriba. Ahora bien, estas técnicas no permiten controlar el compromiso entre la calidad espacial y la espectral de las imágenes fusionadas.

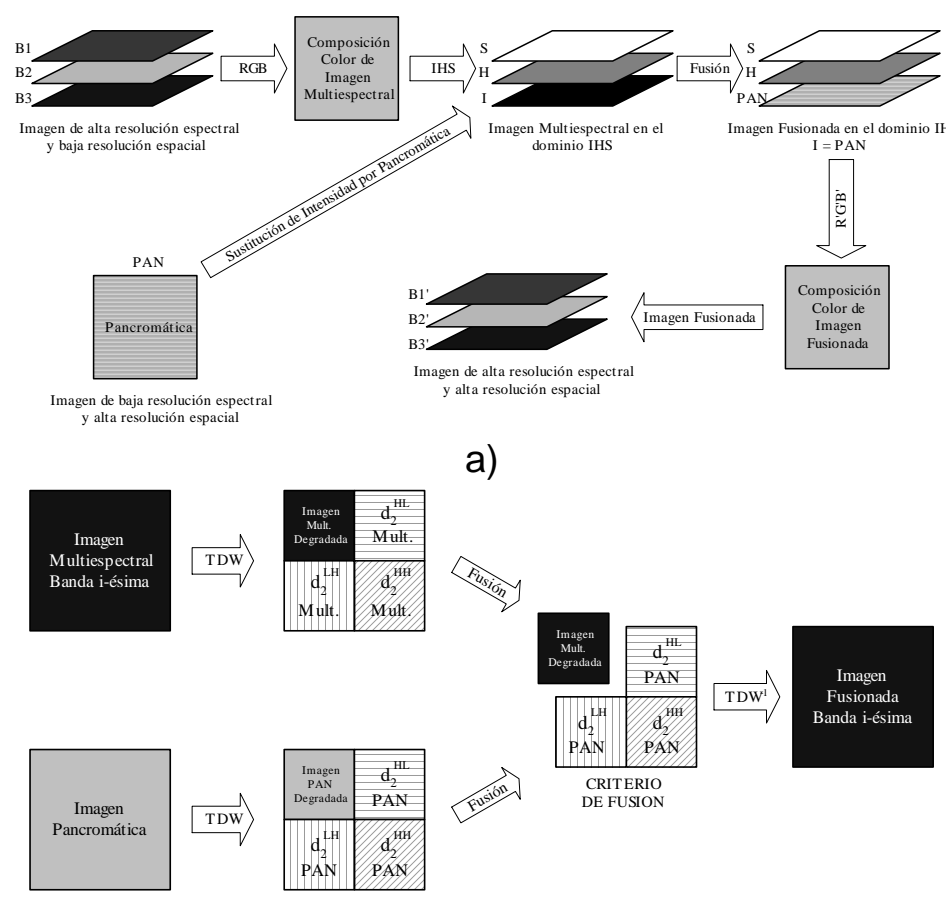

b)

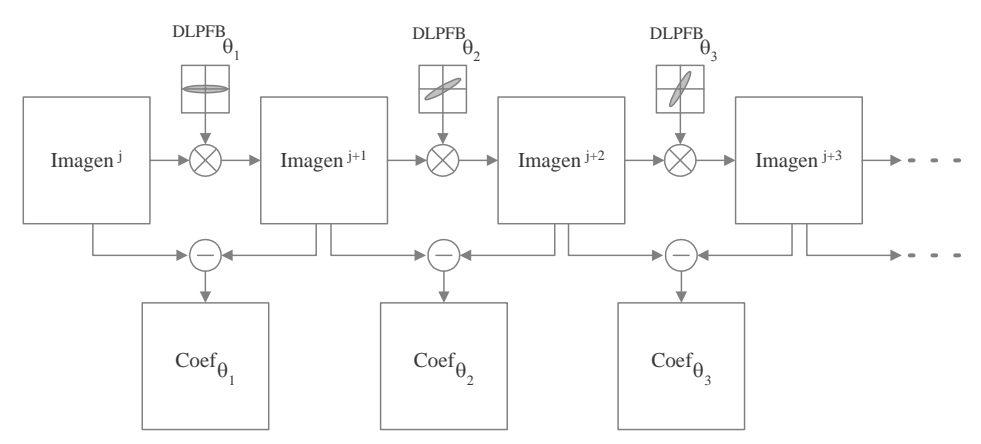

c)

Fig. 1: a) Esquemas del método de fusión basado en la transformada IHS y b) basado en la transformada Wavelet, calculada mediante el algoritmo de Mallat. c) Esquema de separación de la información mediante la transformada MDMR. 
En este sentido Lillo-Saavedra y Gonzalo (2006), propusieron una versión ponderada de la fusión mediante TDW calculada con el algoritmo à trous. Un parámetro $\alpha$ pondera la cantidad de detalle procedente de la imagen pancromática que se inyecta en una versión degradada de la imagen multiespectral, lo que permite el control del compromiso entre las dos características mencionadas. En está misma línea de investigación, Otazu et al. (2005) propusieron un nuevo esquema de fusión, también basado en la TDW, que teniendo en cuenta las curvas de respuesta espectral de los sensores multiespectral y pancromático, determinan la cantidad de detalle espacial de la imagen pancromática que debe incorporarse a cada una de las bandas de la imagen multiespectral. Los resultados obtenidos en este caso dependen fuertemente del grado de solapamiento entre las curvas de respuesta del sensor multiespectral y el pancromático. Estas aproximaciones permiten controlar el compromiso entre la calidad espacial y espectral de las imágenes fusionadas mediante mecanismos externos a las transformadas en las que se basa el método de fusión.

El método de fusión multidirección-multirresolución (MDMR) propuesto por Lillo-Saavedra y Gonzalo (2007), permite el control simultáneo del compromiso entre la calidad espacial y la distorsión del color de dichas imágenes. Este método se basa en un único banco de filtros paso bajo direccional, cuyos parámetros ( $\mathrm{a}$ y b) determinan la cantidad de información que aportan cada una de las imágenes fuente a la imagen final fusionada. En la figura 1 c) se muestra un esquema del proceso de separación de la información de detalle (altas frecuencias espaciales) y de fondo (bajas frecuencias espaciales) mediante la transformada MDMR propuesta por Lillo-Saavedra y Gonzalo (2007), para posteriormente, integrar, mediante un criterio aditivo, el detalle direccional extraído de la pancromática en la imagen multiespectral degradada, también direccionalmente.

Con objeto de mostrar la capacidad de este método para controlar la calidad espacial y espectral, se han obtenido imágenes fusionadas para diferentes valores de los parámetros a y b. Y se ha evaluado la calidad de dichas imágenes mediante el índice de correlación espectral (CC) (Vijayaraj et al., 2004) y el índice de Zhou (Zhou et al., 1998), el cual da una medida de la calidad espacial de la imagen fusionada. Estos índices se obtienen promediando los valores de correlación obtenidos banda a banda, de la imagen fusionada respecto a la imagen MULTI original para el CC y el de Zhou, correlando la imagen fusionada y la PAN filtradas con un filtro Laplaciano. Al ser índices basados en la correlación, su valor ideal es 1.

La Tabla 1 recoge los valores de estos índices para imágenes fusionadas con el método MDMR, para valores de los parámetros del banco de filtros $a=9$ y $b=0.5,1,2,3$ y 4 . Estos valores se han determinado aplicando el criterio mostrado en Lillo-Saavedra y Gonzalo (2007) a la imagen objeto de estudio. Se puede observar que para un valor de a constante $(a=9)$, a medida que el parámetro $b$ aumenta, la calidad espectral de las imágenes fusionadas mejora, empeorando su calidad espacial. Queda por tanto probado la capacidad del método MDMR, para controlar el compromiso entre la calidad espacial y espectral de las imágenes fusionadas, dependiendo de cual sea su aplicación final. En el caso que nos ocupa, la estimación de turbidez en lagos, es necesario un equilibrio entre ambas calidades, por lo que se ha considerado como mejor imagen fusionada la imagen obtenida con los parámetros $a=9$ y $b=2$.

Tabla 1: Índices de calidad de imágenes fusionadas con el método MDMR con diferentes filtros.

\begin{tabular}{cccc}
\hline $\mathrm{a}$ & $\mathrm{b}$ & CC & Zhou \\
\hline 9 & 0,5 & 0,9085 & 0,9985 \\
9 & 1 & 0,9403 & 0,9909 \\
9 & 2 & 0,9762 & 0,9036 \\
9 & 3 & 0,9907 & 0,7193 \\
9 & 4 & 0,9957 & 0,5529 \\
\hline
\end{tabular}

Como una forma de cuantificar la calidad de las imágenes obtenidas mediante el método MDMR frente a otros métodos de fusión, se han obtenido imágenes fusionadas para los tres métodos ilustrados en la figura 1 y se han evaluado los índices CC y de Zhou. Se debe señalar, que dada la limitación del método IHS en cuanto al número de bandas a fusionar, se han evaluado en los tres casos los índices correspondientes a las bandas 2, 3 y 4 de las imágenes fusionadas. Los valores 
obtenidos para las tres imágenes fusionadas, se han incluido en la Tabla 2. Los resultados obtenidos justifican cuantitativamente la utilización del método MDMR en este trabajo.

Tabla 2: Índices de calidad de imágenes fusionadas con diferentes métodos.

\begin{tabular}{ccc}
\hline Método & CC & Zhou \\
\hline IHS & 0.8821 & 0.8964 \\
W-M & 0.9333 & 0.9130 \\
MDMR & 0.9479 & 0.9895 \\
\hline
\end{tabular}

\section{ESTIMACIÓN DE LA TURBIDEZ EN LAGOS A PARTIR DE IMÁGENES DE SATELITE}

Los sólidos en suspensión son los agentes contaminantes más comunes en los sistemas lénticos (lacustres), estos incrementan la intensidad de la radiancia reflejada por la superficie del lago, en el rango visible y en el infrarrojo cercano del espectro electromagnético (Ritchie et al., 2001). La medición de este parámetro se realiza mediante el uso "in situ" de un dispositivo denominado disco "Secchi". Este proceso, limita la estimación en ecosistema léntico, debido a la imposibilidad de disponer de un número suficiente de muestras representativas y adecuadamente distribuidas tanto espacialmente como temporalmente.

Una solución complementaria al problema planteado en el párrafo anterior, es la utilización de técnicas de teledetección para la identificación y evaluación de las variables más representativas para la determinación de la calidad de agua en un lago, en particular la turbidez. Su principal ventaja radica en que esta técnica proporciona una visión sinóptica tanto espacial como temporal del fenómeno bajo observación, variables fundamentales para realizar una mejor gestión y control de los recursos hídricos. Sin embargo, existe una dependencia directa de la calidad y disponibilidad de estas estimaciones con respecto a la resolución tanto espectral como espacial de las imágenes de satélite utilizadas. Es por ello que la obtención de imágenes fusionadas que mejoren estas resoluciones y posibiliten su aplicación a la estimación de variables biofísicas cobra importancia.

Tyler et al. (2006), propusieron la calibración de un modelo paramétrico que relaciona, mediante una regresión de tipo lineal, la banda tres del sensor Landsat (6300 a los $6900 \mu \mathrm{m}$ ), con los datos de profundidad Secchi, obtenidos en una campaña de terreno, con objeto de realizar estimaciones de turbidez. Un aspecto crítico para la correcta calibración del modelo es el diseño adecuado de una estrategia de muestreo de profundidades Secchi. En este sentido Galindo (2005), plantea que cuando se desconoce la caracterización de la distribución espacial de los parámetros a medir en el lago, es recomendable realizar un muestreo de tipo aleatorio con un número de mediciones tal que permita abarcar la mayor superficie posible del lago.

\section{METODOLOGÍA}

Los datos utilizados para evaluar la metodología de fusión y su aplicación en la determinación de la turbidez de un lago, corresponden a una escena de las imágenes registradas por los sensores pancromático y multiespectral del satélite Landsat 7 ETM+ el día 22 de diciembre de 2005 (row:086, path:001). Geográficamente la escena se ubica en la zona de San Pedro de la Paz, Concepción, Chile, La resolución espacial del sensor multiespectral de satélite Landsat 7 ETM+, es de 30m para las bandas del visible e infrarrojo, excepto el térmico, $(1,2,3,4,5$ y 7), siendo la del sensor pancromático de 15m. La figura 2 (a) muestra la banda 3 de la imagen MULTI original. En la parte izquierda de esta escena se localizan la Laguna Grande de San Pedro ( $36^{\circ} 51^{\prime} \mathrm{S}, 73^{\circ} 06^{\prime} 0$ ), que corresponde a la zona de estudio de este trabajo. Las características morfológicas principales de esta laguna son: un área superficial de $155.73 \mathrm{Ha}$; una profundidad máxima de $11.8 \mathrm{~m}$. y una profundidad media de 8.3 m.; altura a nivel del mar de 4 m.s.n.m.

Previamente al proceso de fusión y con objeto de asegurar una perfecta superposición entre ambas imágenes, es preciso remuestrear y co-referenciar la imagen MULTI respecto a la imagen PAN (ambos productos correspondientes a un nivel 5 de corrección). Este preprocesado se realizó con el módulo de co-registro automático de la herramienta ENVI 4.3. 
Aplicando la metodología de fusión MDMR, así como los parámetros de los filtros ya comentados $(a=9, b=2)$, se ha obtenido una imagen multiespectral con una resolución espectral de 6 bandas, idénticas a la imagen MULTI, y con una resolución espacial de $15 \mathrm{~m}$, asociada a la imagen PAN. La implementación de la metodología de fusión MDMR fue realizada íntegramente en la plataforma de desarrollo MATLAB versión 7.0. La banda 3 de la imagen fusionada, mediante la metodología MDMR, se muestra en la figura 2 (b).

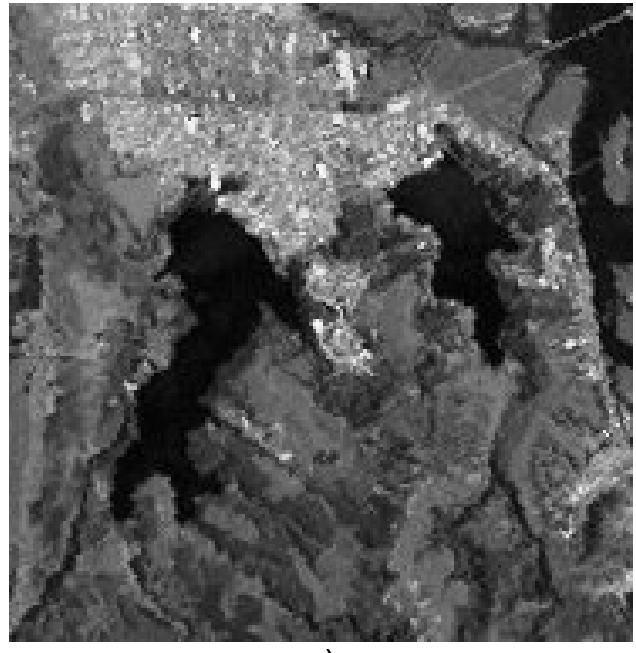

a)

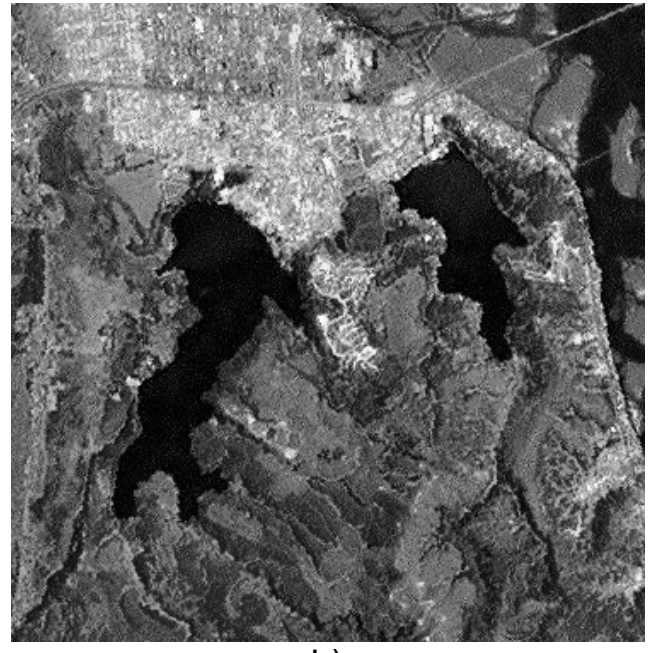

b)

Fig. 2: Banda 3 de las imágenes: a) MULTI y b) Fusionada.

Como se mencionó anteriormente, el parámetro que se ha seleccionado como indicador de la calidad de cuerpos de agua, para llevar a cabo el estudio propuesto, ha sido la profundidad Secchi. Este indicador se midió "in situ", en una campaña de campo realizada el día 15 de diciembre de 2005. Respecto a las zonas de captura de datos, fue necesario realizar una inspección generalizada del lago, con el objetivo de detectar variabilidad en algunas zonas que presentan actividad antropogénica así como notorias variaciones de la velocidad de viento, tal que generen oleaje a nivel de superficie. Esta inspección proporcionó una primera aproximación de las áreas de interés para la toma de datos. A partir de la esta aproximación, se diseñó una metodología de adquisición de datos de tipo aleatoria, que restringió tanto las zonas de captura de los mismos así como la ventana de tiempo en la que se debía realizar. Por un lado, es preciso evitar las neblinas matinales presentes en habitualmente esta zona, por lo cual es recomendable iniciar las medidas a las 11:00 hrs. AM, sin extenderse mas allá de las 13:00 hrs. PM, para que dichas medidas puedan ser consistentes temporalmente con los datos registrados por el sensor remoto. La relación entre el área superficial a muestrear y la ventana de tiempo determina el número de muestras que se pueden medir. Además, cada lectura de la profundidad Secchi, debe ser georeferenciada, para ello se utilizó un GPS portátil Garmin eTrex.

Los valores de profundidad obtenidos por medio del disco Secchi, deben ser agrupados por un criterio de cercanía espacial y depurados mediante un proceso de filtrado, con el objetivo de mejorar la bondad de ajuste de la estimación. Estos valores permiten establecer una relación directa de la cantidad de sólidos en suspensión presentes en las distintas zonas del cuerpo de agua (Olmanson et al. 2002), siendo este uno de los parámetros de evaluación más importante para la determinación del estado trófico de un lago.

El conjunto de datos obtenidos de la campaña de terreno, se relacionan, mediante un ajuste lineal con los valores digitales de la banda 3, geográficamente coincidentes y corregidos mediante el método de los píxeles obscuros, tanto para la imagen MULTI (original) como fusionada, según la metodología propuesta por Tyler et al. (2006).

\section{RESULTADOS Y DISCUSIÓN}

El resultado de aplicar la metodología descrita en el apartado anterior a la imagen MULTI y a la imagen fusionada se muestra en la figura 3 (a) y (b). En esta figura, es posible apreciar un aumento 
del rango de variación de los valores digitales en la banda 3 para el caso de la imagen fusionada, respecto a los valores medidos "in situ", esto queda en evidencia con la disminución de la pendiente de la curva de regresión para la estimación a partir de la imagen fusionada. Consecuentemente, el valor de $r^{2}$ obtenido para el caso de la imagen MULTI (0.8591) es ligeramente superior al obtenido para la imagen fusionada (0.8568). Ello es debido a que la menor resolución de la imagen MULTI permite discriminar un menor número de valores de niveles digitales (ND) y por lo tanto de rangos de profundidad.

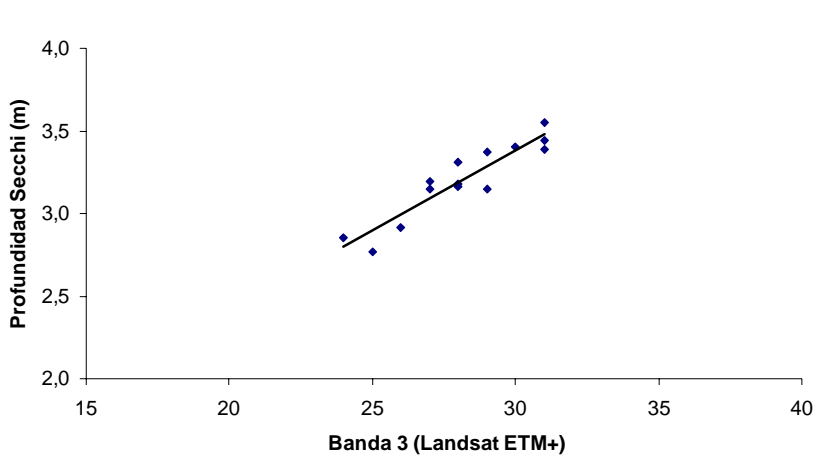

a)

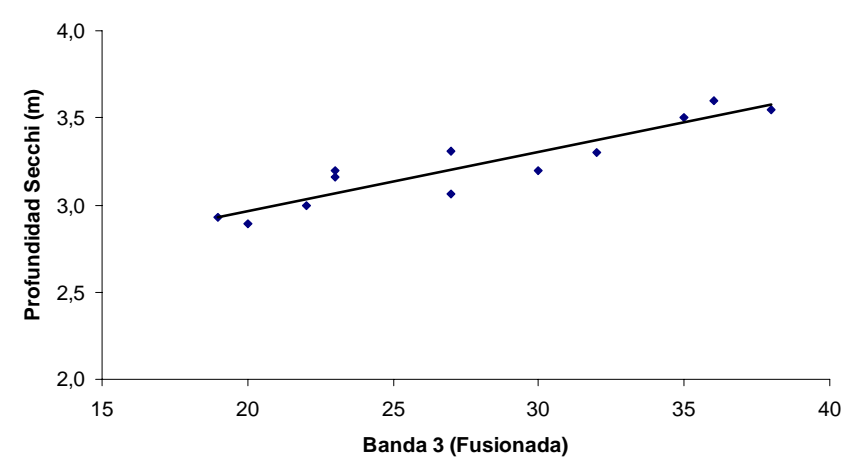

b)

Fig. 3: Regresión lineal entre profundidad Secchi (m) y: a) ETM3 de la imagen original. b) Banda 3 de la imagen fusionada.

Los resultados anteriores se pueden visualizar espacialmente mediante los mapas de distribución de las profundidades Secchi, generados a partir de las ecuaciones de regresión obtenidas para las imágenes MULTI y fusionada respectivamente (1 y 2 ).

Secchi $\left(N D_{\text {MULTI }}\right)=0.0330 \cdot N D_{\text {MULTI }}+2.2926$
Secchi $\left(N D_{\text {Fusionada }}\right)=0.0967 \cdot N D_{\text {Fusionada }}+0.4796$

Donde $N_{\text {MULTI }}$ y $N D_{\text {Fusionada }}$ son los valores de niveles digitales de las imágenes MULTI y fusionada, respectivamente. Los mapas mostrados en las figuras 4 (a) y (b) muestran una versión umbralizada con cinco niveles de los mapas originales. Una comparación visual de los resultados obtenidos muestra que, un aumento de 1:2 en la relación espacial de las imágenes utilizadas, permite una caracterización más precisa de la distribución espacial de los contenidos de sólidos en suspensión presentes en el lago. Esta situación se justifica, principalmente, por el hecho de que un píxel de $30 \mathrm{~m}$ de resolución espacial integra en un solo valor digital una gran cantidad de respuestas espectrales, que caracterizan a diferentes contenidos de sólidos en suspensión. Por el contrario, un píxel de un tamaño menor $(15 \mathrm{~m})$ integrará una menor cantidad de tipos de cubiertas, esto implica píxeles con un contenido espectral de mayor pureza. Por consiguiente, en la imagen fusionada, será posible identificar profundidades Secchi que en la imagen original no era posible detectar.

En la figura 5, se presenta la distribución de los diferentes valores de profundidad Secchi para la imagen original y fusionada. Es posible apreciar que existe un aumento en el rango de valores de profundidad estimado por medio de la imagen fusionada respecto de la imagen original, lo que confirma el resultado visual planteado en el párrafo anterior.

Se puede observar en esta figura la importancia de determinar el estado de eutrofización del lago a partir de imágenes de satélite con diferente resolución espacial. Así para la imagen de 30 m (MULTI) la profundidad predominante está en el rango 1-2 m, mientras que para la fusionada está en el rango de 0-1m., ello muestra que la disponibilidad de imágenes de mayor resolución espacial permite una mejor información para la toma de decisiones en la gestión del lago. 


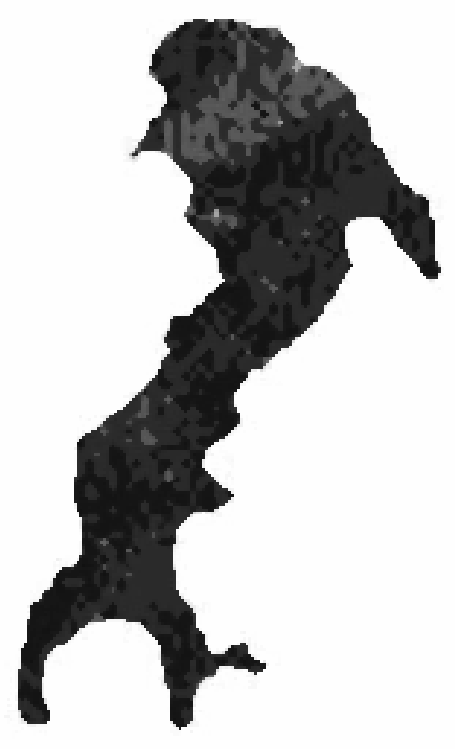

a)
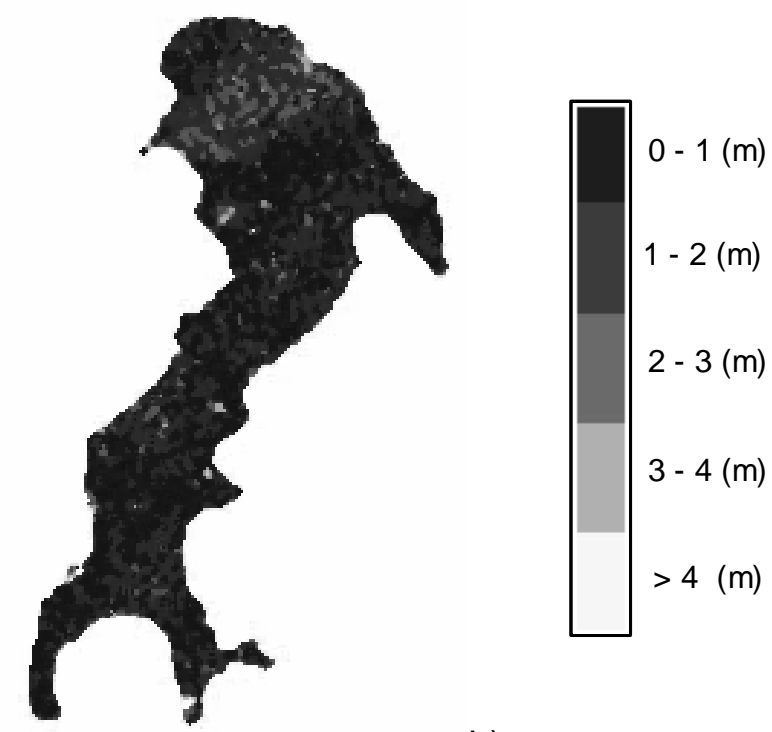

b)

Fig. 4: Mapa de profundidades Secchi obtenidos a partir de los modelos de ajuste lineal para: a) La imagen MULTI (original) y b) imagen fusionada.

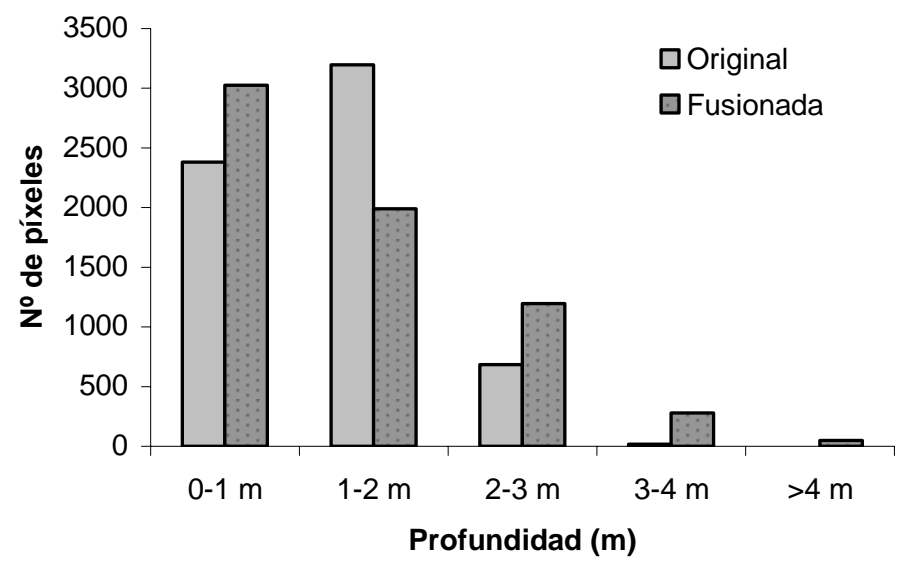

Fig. 5: Distribución de los valores de profundidad Secchi para la imagen original y fusionada

\section{CONCLUSIONES}

En este trabajo se ha mostrado que el método de fusión MDMR permite controlar las características espaciales y espectrales de las imágenes resultantes, mediante la utilización de valores adecuados de los parámetros del banco de filtros asociado a dicho método, adaptando dichas características a su aplicación.

Una imagen multiespectral de baja o media resolución espacial, puede llevar a una caracterización errónea del nivel de turbidez de un lago de pequeña o media escala. En este sentido, los resultados obtenidos en este trabajo permiten concluir que la utilización de imágenes de satélite obtenidas a partir de la fusión de imágenes PAN y MULTI, mediante la metodología MDMR, aumenta el rango dinámico de los valores de ND implicados en la estimación del parámetro biofísico de turbidez, preservando las características espectrales de la imagen MULTI. Ello ha permitido una mejor discriminación de estos valores. Además la mayor resolución espacial de estas imágenes proporciona una mejora en su distribución espacial. Por lo que este tipo de imágenes se presentan como una buena alternativa, sin costo económico añadido, para ayudar a la toma de decisiones en la gestión del agua en los sistemas lénticos. 


\section{AGRADECIMIENTOS}

Este trabajo se ha llevado a cabo bajo la financiación de los proyectos: FONDECYT \#11060056, del Consejo Nacional de Ciencia y Tecnología de Chile, TEC2007-60607/TCM del Ministerio de Educación y Ciencia de España y AL08-P(I+D)-19 de la Universidad Politécnica de Madrid.

\section{REFERENCIAS}

Choi, M.; A New Intensity-Hue-Saturation Fusion Approach to Image Fusion with a Tradeoff Parameter. IEEE Transactions on Geoscience and Remote Sensing: 44, 1672-1682 (2006).

Do, M. y M. Vetterli; The Contourlet Transform: an Efficient Directional Multiresolution Image Representation. IEEE Transactions on Image Processing: 14, 2091-2106 (2005).

Galindo, M.; Water Quality and Spatial Variability in Lake Cutizeo, México. Master thesis submitted to the International Institute for Geo-Information Sciences and Earth Observation (2005).

Garzelli, A., F. Nencini y L. Alparone; Pan-Sharpening of Multispectral Images: A Critical Review and Comparison, IEEE International Geoscience and Remote Sensing Symposium, 81-84 (2004).

Gónzalez-Audicana, M., J. L. Saleta, R. García Catalán y R. García; Fusion of Multiespectral and Panchromatic Images Using Improved IHS and PCA Mergers based on Wavelet Decomposition, IEEE Transactions on Geosciences and Remote Sensing: 42 (6), 1291-1297 (2004).

González-Audicana, M., X. Otazu, O. Fors y A. Seco; Comparison Between the Mallat's and the à trous Discrete Wavelet Transform Based Algorithms for the Fusion of Multispectral and Panchromatic Images, International Journal of Remote Sensing; 26: 597-616 (2005).

Gonzalo, C. y M. Lillo-Saavedra; Fusión de Imágenes Quickbird Mediante una Representación Conjunta Multirresolución-Multidirección. IEEE Latin America Transaction: 5(1) 32-35 (2007).

Lillo-Saavedra M. y C. Gonzalo; Spectral or Spatial Quality for Fused Satellite Imagery? A Trade-Off Solution Using Wavelet à trous Algorithm. International Journal of Remote Sensing: 27(7), 1453-1464 (2006).

Lillo-Saavedra, M. y C., Gonzalo; Multispectral Images Fusion by a Joint Multidirectional and Multiresolution Representation. International Journal of Remote Sensing: 28(18), 4065-4079 (2007). Mallat, S.; A Wavelet Tour of Signal Processing, 2nd ed., Academic Press, Elsevier (1999).

Núñez, J. y otros seis autores; Multiresolution-Based Image Fusion with Additive Wavelet Decomposition. IEEE Transactions on Geoscience and Remote Sensing: 37, 1204-1211 (1999).

Olmanson, L., M. Bauer y P., Brezonik; Water Quality Monitoring of 10000 Minnesota Lakes: Statewide Classification of Lake Water Clarity Using Landsat Imagery. Proceedings, 15th Willian $T$. Pecora Memorial Remote Sensing Symposium. Denver, Colorado (2001).

Otazu, X., M. González-Audicana, O. Fors y J. Nuñez; Introduction of Sensor Spectral Response into Fusion Methods. Application to Wavelet Methods, IEEE Transactions on Geosciences and Remote Sensing: 43(10), 2376-2385 (2005).

Ritchie, J., S. Seyfried, M., Chopping e Y., Pachepsky; Airborne Laser Technology for Measuring Rangeland Conditions. Journal of Range Manage: 54(2), A8-A21 (2001).

Tu, T.-M., S.-C. Su, , H.-Ch. Shyu y P.S. Huang; A New Look at IHS-Like Image Fusion Methods, Information Fusion: 2, 177-186 (2001).

Tyler , A. y otros cuatro autores; A Remote Sensing of the Water Quality Shallow lakes: A Mixture 
Modelling Approach to Quantifying Phytoplankton in Water Characterized by High-Suspended Sediment. International Journal of Remote Sensing: 27(8), 1521-1537 (2006).

Wald, L.; Data Fusion, Defibçnition and Architectures: Fusion of Image of Different Spatial Resolution, Le Presses de l'Ecole des Mines, Paris (2002).

Vijayaraj, V., Ch., O'Hara y N., Younan; Quality Analysis of Pan-Sharpened Images. Proceedings of the Geosciences and Remote Sensing Symposium (IGARSS), 20-24 September 2004, Anchorage, Alaska , 85-88 (2004).

Zhou, J., D. Civco y J. Silander; A Wavelet Method to Merge Landsat TM and SPOT Panchromatic Data. International Journal of Remote Sensing: 19, 743-757 (1998). 